\title{
Versatile regularisation toolkit for iterative image reconstruction with proximal splitting algorithms
}

Daniil Kazantsev, Edoardo Pasca, Mark Basham, Martin Turner, Matthias J. Ehrhardt, et al.

Daniil Kazantsev, Edoardo Pasca, Mark Basham, Martin Turner, Matthias J. Ehrhardt, Kris Thielemans, Benjamin A. Thomas, Evgueni Ovtchinnikov, Philip J. Withers, Alun W. Ashton, "Versatile regularisation toolkit for iterative image reconstruction with proximal splitting algorithms," Proc. SPIE 11072, 15th International Meeting on Fully Three-Dimensional Image Reconstruction in Radiology and Nuclear Medicine, 110722D (28 May 2019); doi: $10.1117 / 12.2534289$ Nuclear Medicine, 2019, Philadelphia, United States 


\title{
Versatile regularisation toolkit for iterative image reconstruction with proximal splitting algorithms
}

\author{
Daniil Kazantsev ${ }^{\mathrm{a}, *}$, Edoardo Pasca ${ }^{\mathrm{b}}$, Mark Basham ${ }^{\mathrm{a}}$, Martin Turner ${ }^{\mathrm{b}}$, Matthias J. \\ Ehrhardt $^{\mathrm{c}}$, Kris Thielemans ${ }^{\mathrm{d}}$, Benjamin A. Thomas ${ }^{\mathrm{d}}$, Evgueni Ovtchinnikov ${ }^{\mathrm{b}}$, Philip J. \\ Withers $^{\mathrm{e}}$, Alun W. Ashton ${ }^{\mathrm{a}}$ \\ ${ }^{a}$ Diamond Light Source Ltd., Scientific Software Department, Diamond House, Harwell Science \& Innovation \\ Campus, Didcot, UK, OX11 0DE \\ ${ }^{\mathrm{b}}$ Scientific Computing Department, STFC, Rutherford Appleton Laboratory, Didcot, UK, OX11 0QX \\ ${ }^{c}$ Institute for Mathematical Innovation, University of Bath, Bath, UK, BA2 7JU \\ ${ }^{\mathrm{d}}$ Institute of Nuclear Medicine, UCL, London, UK, NW1 2BU \\ ${ }^{\text {e}}$ The Henry Royce Institute, School of Materials, The University of Manchester, UK, M13 9PL
}

\begin{abstract}
Ill-posed image recovery requires regularisation to ensure stability. The presented open-source regularisation toolkit consists of state-of-the-art variational algorithms which can be embedded in a plug-and-play fashion into the general framework of proximal splitting methods. The packaged regularisers aim to satisfy various prior expectations of the investigated objects, e.g., their structural characteristics, smooth or non-smooth surface morphology. The flexibility of the toolkit helps with the design of more advanced model-based iterative reconstruction methods for different imaging modalities while operating with simpler building blocks. The toolkit is written for CPU and GPU architectures and wrapped for Python/MATLAB. We demonstrate the functionality of the toolkit in application to Positron Emission Tomography (PET) and X-ray synchrotron computed tomography (CT).
\end{abstract}

Keywords: X-ray CT, PET, iterative methods, model-based, proximal-dual, regularization.

* The corresponding author daniil.kazantsev@ diamond.ac.uk

\section{Introduction}

In tomography, ${ }^{1}$ various mathematical reconstruction algorithms are applied to the measured projection data to obtain a volume. When the measured projection data is corrupt, noisy and/or angularly undersampled, the inversion is ill-posed and regularised iterative methods (RIM) should be used. A suitable regulariser can be chosen according to some prior expectations about the reconstructed object. For instance, in material science the reconstructed object is frequently piecewiseconstant while in medical imaging piecewise-smooth.

One of the main disadvantages of using RIM for tomographic reconstruction is the computational cost involved in optimising the objective function which consists of the data fidelity and regularisation terms (see Fig. 1). When the terms of the objective function are differentiable, gradient or Hessian-based optimisation methods can be employed. The smoothness constraint, however, might not always be a desirable feature of the objective and one needs to resort to non-smooth optimisation strategies.

The framework of proximal splitting operators (PSO) $)^{2}$ can be applied to indifferentiable cost functions and allows the objective decoupling resulting in simpler, frequently parallelisable, optimisation steps. This enables fast prototyping and implementation of novel RIMs. ${ }^{3}$

In this paper, we introduce the CCPi-Regularisation Toolkit (CCPi-RGL) ${ }^{4}$ for effective and efficient regularisation of iterative methods for application the reconstruction of large 3D tomographic datasets. We demonstrate how the CCPi-RGL toolkit can be used to reconstruct positron emission tomography (PET) data with the STIR-SIRF package ${ }^{5,6}$ and also high-resolution synchrotron tomographic data using MPI-based Savu software. ${ }^{7}$ 


\section{CCPi-RGL software contents and methodology}

The CCPi-RGL software contains various state-of-the-art variational regularisation techniques which include a second and fourth-order diffusion-based methods as well as local and non-local approaches. We refer the reader to the dedicated paper $^{4}$ where all available methods we describe in detail and benchmarks provided to help with method selection.

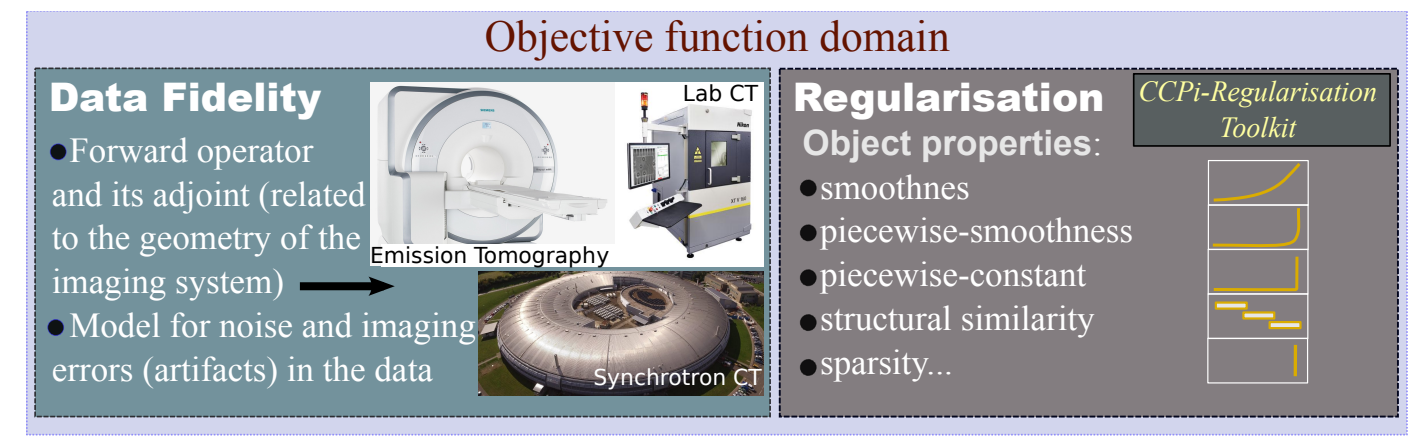

Fig $1 \mathrm{CCP} i-R G L$ within the general optimisation framework.

The general optimisation form for tomographic image reconstruction can be formulated as:

$$
\min _{\boldsymbol{x} \in \mathbb{R}^{N}} \mathcal{F}(\boldsymbol{x})+g(\boldsymbol{x}) \equiv f(\boldsymbol{A} \boldsymbol{x})+g(\boldsymbol{x}) \equiv \sum_{i=1}^{n} f_{i}\left(\boldsymbol{A}_{i} \boldsymbol{x}\right)+g(\boldsymbol{x}),
$$

where $f_{i}: \mathbb{R}^{M_{i}} \rightarrow \mathbb{R}, f: \mathbb{R}^{M} \rightarrow \mathbb{R}$ is a continuously differentiable convex function with Lipschitz continuous gradient. Thus, $\mathcal{F}$ also has Lipschitz continuous gradient and we denote its constant by $L$. The functions $f_{i}$ measure the fidelity of $\boldsymbol{A} \boldsymbol{x}$ to the projection data $\boldsymbol{b} \in \mathbb{R}^{M}$ where $\boldsymbol{A}=$ $\left(\boldsymbol{A}_{1} ; \ldots ; \boldsymbol{A}_{n}\right) \in \mathbb{R}^{M \times N}$ is the linear forward operator and $\boldsymbol{x} \in \mathbb{R}^{N}$ is the unknown solution. The regularisation term $g: \mathbb{R}^{N} \rightarrow \mathbb{R}$ is a convex, possibly non-differentiable function expressing a prior knowledge of the unknown estimate $\boldsymbol{x}$ (see Fig. 1 (left)). In X-ray CT, the common choice for the data fidelity term is the Least-Squares (LS) model: $f(\boldsymbol{A} \boldsymbol{x})=\|\boldsymbol{A} \boldsymbol{x}-\boldsymbol{b}\|_{2}^{2}$ or the Penalised Weighted Least Squares (PWLS): $f(\boldsymbol{A} \boldsymbol{x})=\|\boldsymbol{A} \boldsymbol{x}-\boldsymbol{b}\|_{\boldsymbol{W}}^{2}$, where $\boldsymbol{W} \in \mathbb{R}^{M \times M}$ is a diagonal matrix such as $\left\{W_{i i}=1 / \sigma_{i}^{2}\right\}_{i=1}^{M}$ and $\sigma_{i}^{2} \approx y_{i}^{2}$ is the variance of the measurements. It is not uncommon, especially for emission tomography, ${ }^{5}$ to use the more realistic non-linear Poisson model $f(\boldsymbol{A} \boldsymbol{x})=\langle\boldsymbol{y}, \boldsymbol{A} \boldsymbol{x}\rangle+\langle\boldsymbol{z} \exp (-\boldsymbol{A} \boldsymbol{x}), \mathbf{1}\rangle$.

In order to solve problem (1) efficiently, we rely on the theory of the proximal methods ${ }^{2}$ which helps to split the problem into parts which are easier to solve. Before presenting various splitting approaches, we introduce the notion of the proximal operator:

$$
\operatorname{prox}_{\tau g}(\boldsymbol{u})=\min _{\boldsymbol{x} \in \mathbb{R}^{N}} g(\boldsymbol{x})+\frac{1}{2 \tau}\|\boldsymbol{x}-\boldsymbol{u}\|^{2} .
$$

All regularisation algorithms of CCPi-RGL aim to solve (2) and therefore one needs to be concerned only with the $f$-related sub-problem which is specific to the imaging modality.

Here we present some PSO-based algorithms in which CCPi-RGL can be easily integrated. ${ }^{4}$ A well-known FISTA algorithm ${ }^{8}$ (see Alg. 1) can ensure $O\left(1 / k^{2}\right)$ rate of convergence. FISTA requires $f$ in (1) to be Lipschitz differentiable and include only one proximal step on each iteration 


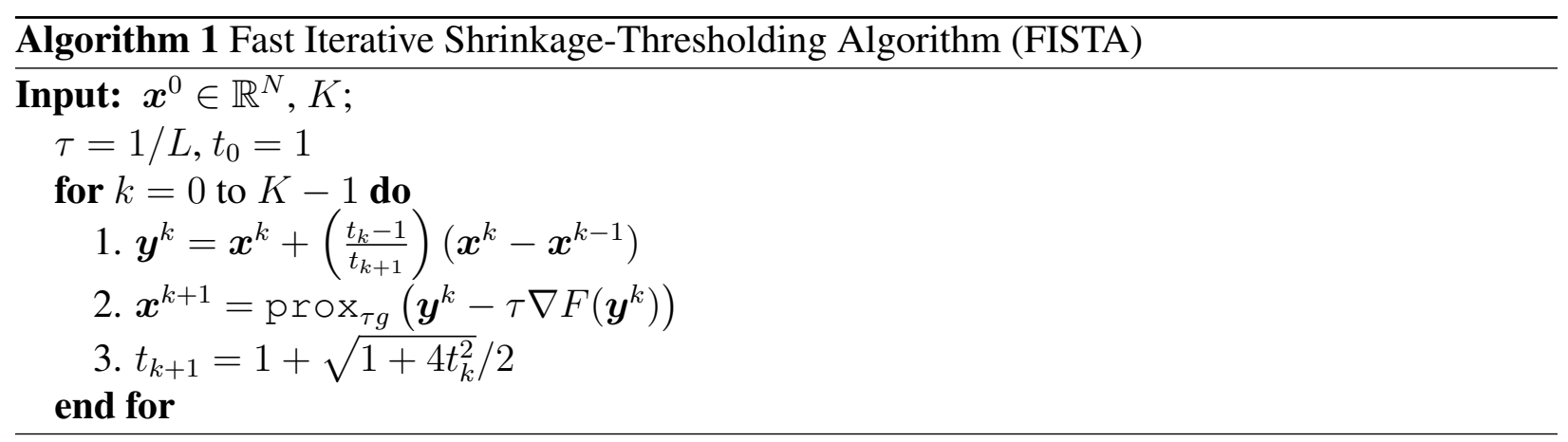

(step 2). The group of primal-dual methods ${ }^{9}$ relax the differentiability condition but normally rely on two proximal steps instead (see Alg. 2).

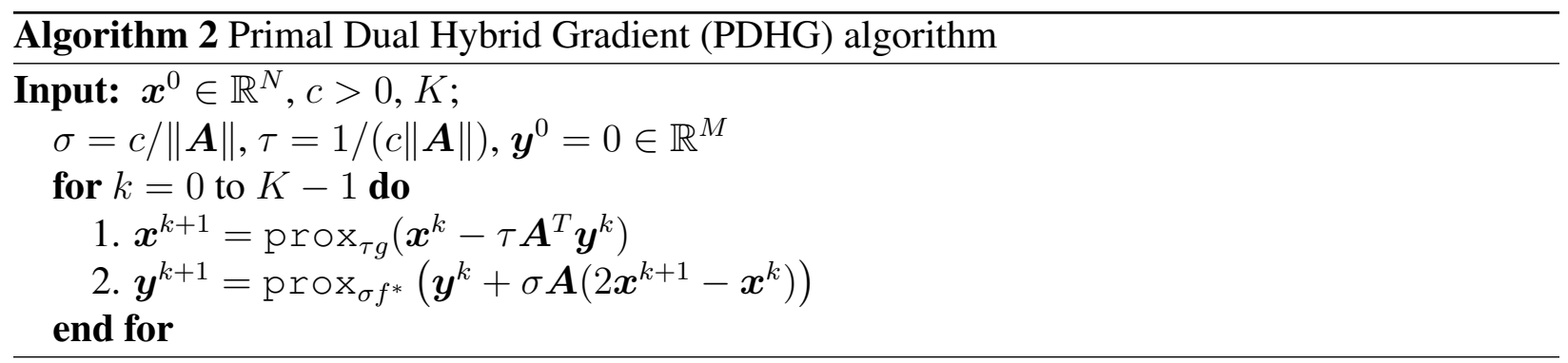

Evaluation $\boldsymbol{A}$ or $\nabla F=\boldsymbol{A}^{\top} \circ \nabla f \circ \boldsymbol{A}$ in each iteration is cumbersome. An approach to overcome this drawback is randomisation which can be achieved within PDHG by selecting only a few dual variables $\boldsymbol{y}_{i}$ in each iteration, resulting in the stochastic $\mathrm{PDHG}^{10,11}$ method (see Alg. 3).

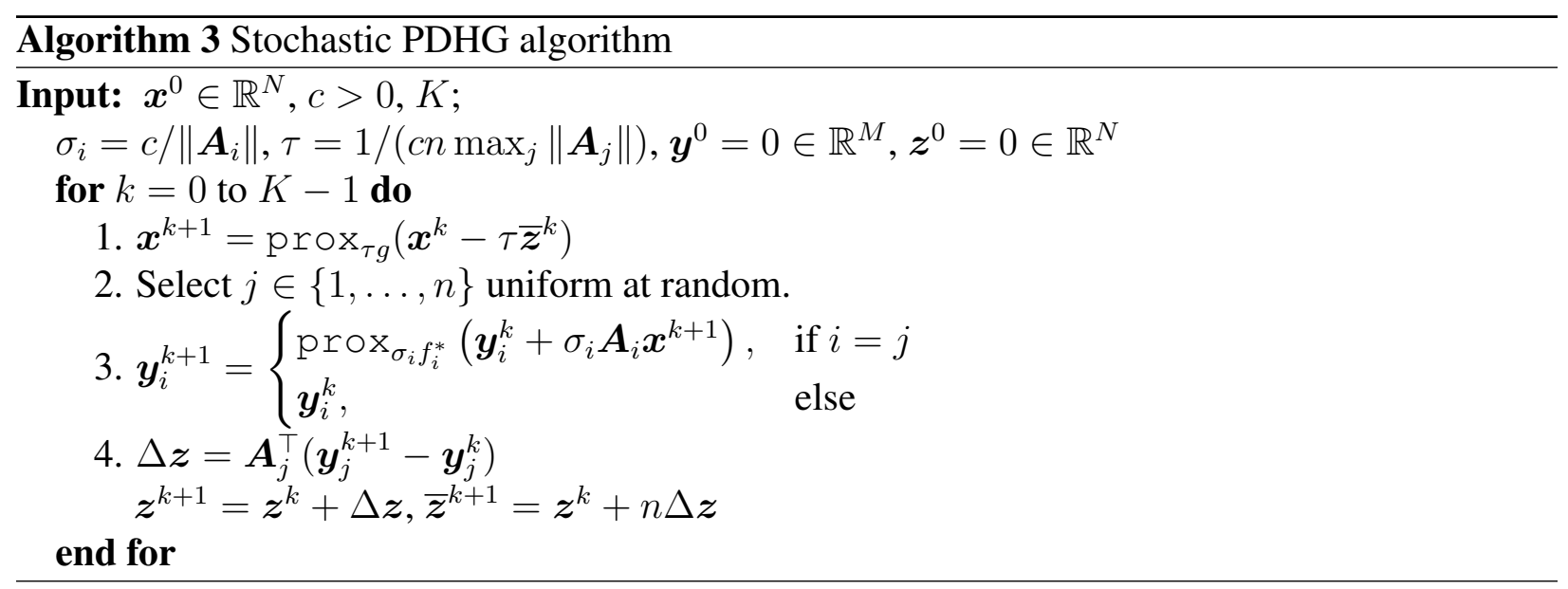

Under the linearisation conditions, the PDHG method becomes the well-known ADMM method ${ }^{2}$ (see Alg. 4). Step 1. of the Alg. 4 is a quadratic optimisation problem when the data fidelity is chosen to be PWLS: $F(\boldsymbol{x})=1 / 2\|\boldsymbol{A} \boldsymbol{x}-\boldsymbol{b}\|_{\boldsymbol{W}}^{2}$. Therefore one needs to solve: $\boldsymbol{x}^{k+1}=$ $\left(\boldsymbol{I}+\tau \boldsymbol{A}^{\top} \boldsymbol{W} \boldsymbol{A}\right)^{-1}\left(\tau \boldsymbol{A}^{\top} \boldsymbol{W} \boldsymbol{b}+\boldsymbol{v}^{k}-\boldsymbol{u}^{k}\right)$ for which Krylov-type methods or Newton solvers can be used. 


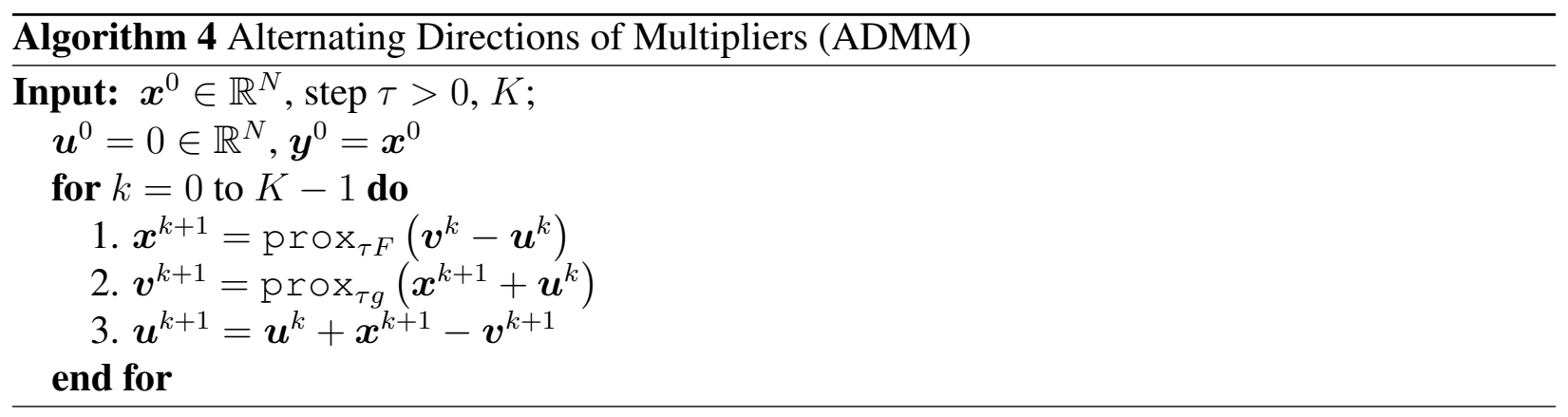

\section{Iterative reconstruction of tomographic data using the CCPi-RGL toolkit}

Here we briefly demonstrate the usage of the CCPi-RGL toolkit applied to tomographic data of two different imaging modalities. Due to the limited space, the main aim is not to quantify or qualify the results, but rather to demonstrate the applicability of CCPi-RGL to different settings.

Our first application is to reconstruct Positron Emission Tomography (PET) data using the $\mathrm{SPDHG}^{10}$ algorithm with the Kullback-Leibler (Poisson) data model and two different regularisers from CCP i-RGL: FGP-TV ${ }^{8}$ and TGV. ${ }^{12}$ We use STIR-SIRF ${ }^{5,6}$ software to model realistic PET system blur and apply Poisson noise to the projection data generated from the thorax phantom (see Fig. 2). We run 50 iterations of the SPDHG algorithm and see that the reconstruction without
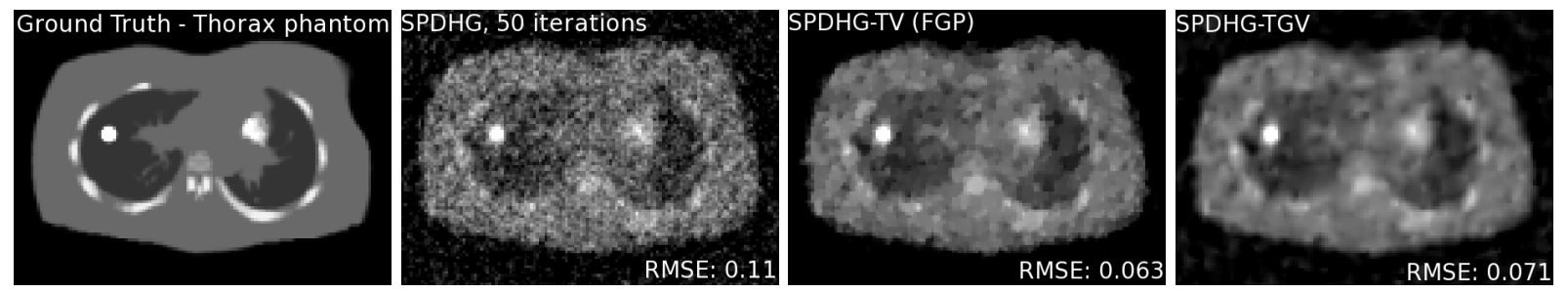

Fig 2 The SPDHG reconstructions of simulated PET data using no regularisation, FGP-TV, and TGV regularisers.

the regularisation is very noisy. Noise can be damped significantly by using regularisation. The higher-order priors, such as TGV might be more preferable than piecewise-constant TV models for emission tomography.

In Fig. 3, we apply CCP i-RGL to reconstruct time-resolved (4D) data of ice-cream under in
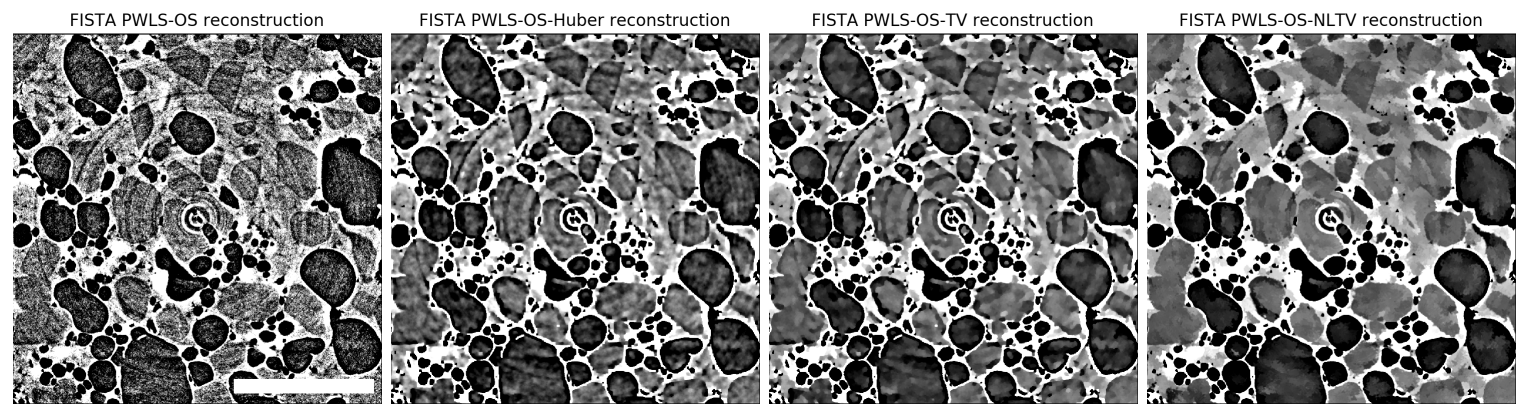

Fig 3 Cropped $1 \mathrm{k}^{2}$ pixels axial slice of reconstructions of $2.5 \mathrm{k}^{3}$ voxels volume using Huber (smoothed TV), FGP-TV and NLTV ${ }^{13}$ regularisers with FISTA PWLS-OS method (12 iterations). The scale bar corresponds to $100 \mu \mathrm{m}$. 
situ thermal cycling conditions ${ }^{14}$ collected at Diamond Light Source (DLS). We use the orderedsubsets accelerated FISTA reconstruction method (see Alg. 1) with the PWLS data model. Qualitatively the NLTV $^{13}$ regulariser outperforms its local rivals.

Additionally, we reconstructed dynamic alloy solidification data also obtained at DLS. ${ }^{15}$ We use the ADMM algorithm (see Alg. 4) with the SB-TV ${ }^{16}$ regulariser. It is evident that the regularised reconstruction can produce much improved images in terms of signal-to-noise ratio and resolution compare to direct techniques (Filtered Backprojection) or unregularised iterative methods.

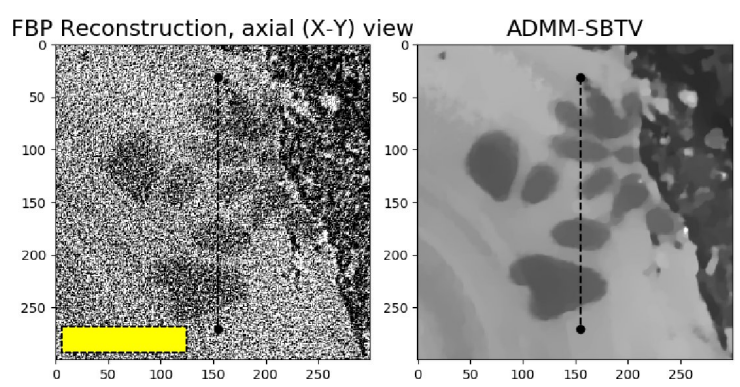

Fig 4 Magnified region of a reconstructed $1 \mathrm{k}^{3}$ voxels volume from the $3 \mathrm{D}$ projection data. Scale bar corresponds to $200 \mu \mathrm{m}$.

\section{Conclusions and acknowledgments}

The presented open-source software CCPi-RGL can be used for tomographic image reconstruction across different imaging modalities. A selection of regularisers provides a desirable customisation and abstraction for fast prototyping of novel reconstruction algorithms. Due to its computationaly efficient implementation, the developed methods can be applied to big data problems across various disciplines.

This work has been partly funded by the Collaborative Computational Project (CCP) in Tomographic Imaging (CCPi) (EP/M022498/1, EP/P02226X/1) and CCP PET-MRI

(EP/M022587/1). The authors acknowledge facilities and the support provided by the Research Complex at Harwell and Diamond Light Source. This work made use of computational support by $\mathrm{CoSeC}$, the Computational Science Centre for Research Communities, through CCPi and CCP PET-MR.

References

1 T. M. Buzug, Computed Tomography: From Photon Statistics to Modern Cone-Beam CT, Springer, Berlin, Germany (2008).

2 A. Chambolle and T. Pock, "An introduction to continuous optimization for imaging," Acta Numerica 25, 161-319 (2016).

3 S. V. Venkatakrishnan, C. A. Bouman, and B. Wohlberg, "Plug-and-play priors for model based reconstruction," in IEEE In Global Conference on Signal and Information Processing, 945-948, IEEE (2013).

4 D. Kazantsev, E. Pasca, M. Turner, et al., "Ccpi-regularisation toolkit for computed tomographic image reconstruction with proximal splitting algorithms," SoftwareX accepted, - (2019).

5 K. Thielemans, C. Tsoumpas, S. Mustafovic, et al., "Stir: software for tomographic image reconstruction release 2," Physics in Medicine \& Biology 57(4), 867 (2012).

6 E. Ovtchinnikov, D. Atkinson, C. Kolbitsch, et al., "Sirf: Synergistic image reconstruction framework," in 2017 IEEE Nuclear Science Symposium and Medical Imaging Conference (NSS/MIC), 1-3, IEEE (2017).

7 N. Wadeson and M. Basham, "Savu: A python-based, mpi framework for simultaneous processing of multiple, n-dimensional, large tomography datasets," arXiv preprint arXiv:1610.08015 (2016).

8 A. Beck and M. Teboulle, "A fast iterative shrinkage-thresholding algorithm for linear inverse problems," SIAM journal on imaging sciences 2(1), 183-202 (2009).

9 A. Chambolle and T. Pock, "A first-order primal-dual algorithm for convex problems with applications to imaging," Journal of mathematical imaging and vision 40(1), 120-145 (2011).

10 A. Chambolle, M. J. Ehrhardt, P. Richtarik, et al., "Stochastic primal-dual hybrid gradient algorithm with arbitrary sampling and imaging applications," SIAM Journal on Optimization 28(4), 2783-2808 (2018).

11 M. J. Ehrhardt, P. Markiewicz, and C.-B. Schönlieb, "Faster pet reconstruction with non-smooth priors by randomization and preconditioning," arXiv preprint arXiv:1808.07150 (2018).

12 K. Bredies, K. Kunisch, and T. Pock, “Total generalized variation,” SIAM Journal on Imaging Sciences 3(3), 492-526 (2010).

13 A. Elmoataz, O. Lezoray, and S. Bougleux, "Nonlocal discrete regularization on weighted graphs: a framework for image and manifold processing," IEEE Trans. Image Processing 17(7), 1047-1060 (2008).

14 E. Guo, D. Kazantsev, J. Mo, et al., "Revealing the microstructural stability of a three-phase soft solid (ice cream) by $4 \mathrm{~d}$ synchrotron x-ray tomography," Journal of Food Engineering 237, 204-214 (2018).

15 D. Kazantsev, E. Guo, A. Phillion, et al., "Model-based iterative reconstruction using higher-order regularization of dynamic synchrotron data," Measurement Science and Technology 28(9), 094004 (2017).

16 T. Goldstein and S. Osher, "The split bregman method for 11-regularized problems," SIAM journal on imaging sciences 2(2), 323-343 (2009). 